\title{
Production of Vortex Beam Modes from a Magnetic Spiral Phase Plate
}

\author{
Arthur M. Blackburn ${ }^{1}$, James C. Loudon ${ }^{2}$ \\ 1. Hitachi Cambridge Laboratory, Cavendish Laboratory, Cambridge, United Kingdom \\ 2. Department of Materials Science and Metallurgy, Cambridge University, Cambridge, United Kingdom
}

Electron vortex beams, where the electron wave front describes a helical path about the beam centre, giving the beam an orbital angular momentum, have so far been produced using diffractive effects involving the beam passing through micro-fabricated gratings with either a fork-like dislocation [1] or a spiral form [2] to yield vortex modes off or on the optical axis respectively. The produced focused beams allow the probing of the magnetic properties of materials at high-resolution [1-3]. In this work, we consider using the stray magnetic field produced from a ferromagnetic rod magnetized along its long axis to create a phase plate (Fig 1a), which in turn is capable of forming a vortex beam.

Micromagnetic modeling of ferromagnetic rods and their associated stray field, show that rods with a suitable geometry can produce a phase shift in the wave function of passing charged particles that approximately describes a helix in the regions near its free ends (Fig. 1a). Placing this rod at the back focal plane of a transmission electron microscope (TEM) (Fig. 1b), with its free end slightly offset $(\sim 100$ $\mathrm{nm}$ ) from the unscattered electron beam creates a phase plate with a spiral-like phase contrast transfer function (Fig. 1c). This plate thus offers some advantages for imaging weak phase objects, and also allows the production of vortex beam modes on-axis.

Calculated point spread functions for example cases with the rod containing a multiple $(n)$ of the fundamental magnetic flux, i.e. $n h / e$, reveals that in the case where $n=1$, the point spread function (PSF) intensity has an annular form (Fig. 2a), with a slight deformation due to the non-perfect spiral phase formed from the rod stray-field. When $n=1.5$, a composite mode appears (Figs. 2c, e), whereas when $n=2$ (or a higher near-integer $n$ ), a single vortex mode dominates (Fig. 2b). With $n=2.35$, as used in our first experiments, a composite mode again appears (Fig. 2d, f), though with the secondary vortex loop having a lower (factor of $\sim 0.1$ ) peak intensity than the primary loop.

Looking over a wider region in the PSF, we see the consequence of electrons passing through (from the classical perspective) the ferromagnetic rod: a diffraction peak with its center perpendicularly offset from the long axis of the rod. As the rod occupies only a small portion of the phase plate aperture, the intensity of this peak is typically more than 3 orders of magnitude less than that of the central peak. Experimental observation of the diffraction pattern formed from a 50Co50Fe rod of length $125 \mu \mathrm{m}$ having a cross section containing a flux of approximately (from holography) $2.35 \mathrm{~h} / \mathrm{e}$, positioned with one end near the center of a $40 \mu \mathrm{m}$ diameter opening (Fig. $2 \mathrm{~g}$ ) confirms the form of the calculated wide area PSF. The central region of the observed pattern, saturated in Fig. 2g, is magnified using a Gatan Imaging Filter attached to the used Philips CM300 TEM, yielding details of the PSF centre (Fig. 2h).

This shows a form similar to that expected from Fig. 2d, though with an aberration that is reminiscent of three-fold astigmatism. Including spherical, chromatic and three-fold astigmatism aberrations in the PSF calculation, convolving this with the demagnified source image and including some misalignment of the rod, goes someway towards creating a probe model (Fig. 2i) that agree with experiment. However, it appears that other aberrations need to be considered too. Most dominant among these is the effect of 
charging up of the carbon coated silicon nitride membrane upon which the $50 \mathrm{Co} 50 \mathrm{Fe}$ rod is supported, which creates additional aberrations. Further, in this first experiment the membrane is positioned a few millimeters above a limiting aperture, and so is not integrated with the aperture as shown in Fig. $1 \mathrm{~b}$. Thus, secondary electrons emitted from the aperture impact the membrane and its silicon carrier over a large area, worsening charge up effects and maybe helping contribute to the observed triangular form.

Nonetheless, agreement between the models and observations appears fair and this is likely the first demonstration of a vortex beam mode simply produced from a ferromagnetic rod. Future work will look at integrating the rod and aperture to reduce charge up effects, thus allowing clearer observations of the predicted vortex modes and perhaps opening the way to more simply produced vortex beams.

\section{References:}

[1] J. Verbeeck, H. Tian, and P. Schattschneider, Nature 467 (2010), p. 301

[2] J. Verbeeck, H. Tian, and A. Béché, Ultramicroscopy 113 (2012), p. 83

[3] J. C. Idrobo and S. J. Pennycook, J. Electron. Microsc. 60 (2011), p. 295

(a)

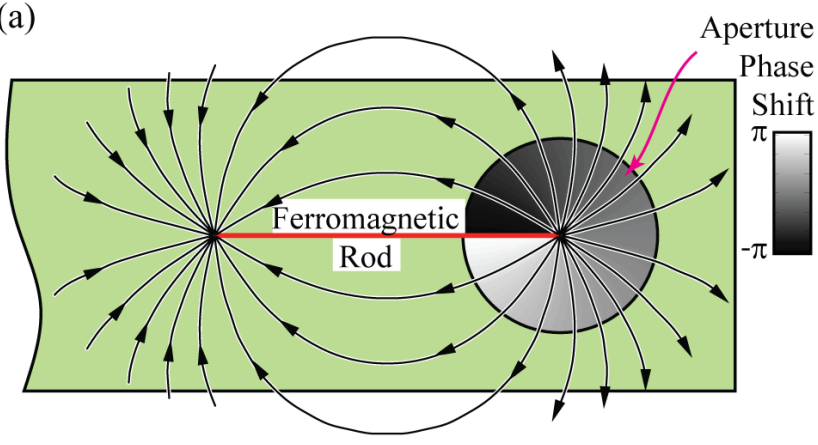

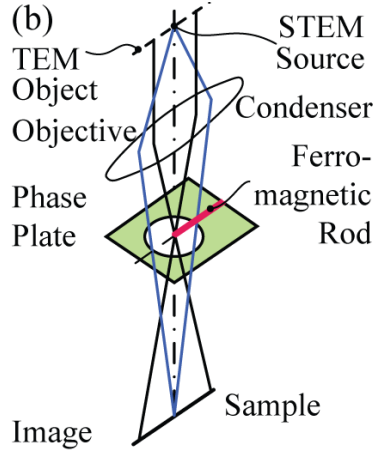

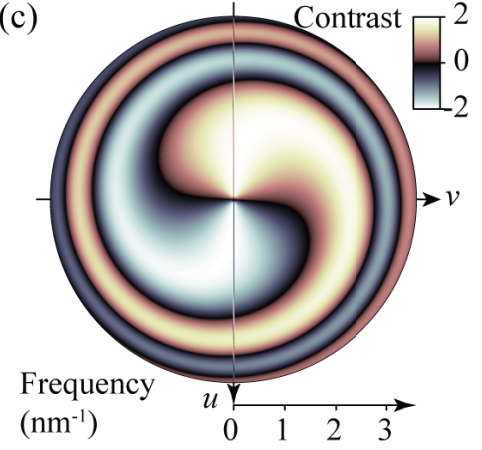

Figure 1. A magnetic rod forms a phase plate (a) that produces an approximately helical phase shift. Positioning this at the condenser aperture position of a STEM (b, right) or back-focal plane of a TEM (b, left), allows the production of a vortex beam or enhancement of TEM imaging of weak phase objects. The phase contrast transfer function (c) is spiral like, given for a typical $100 \mathrm{keV}$ TEM.
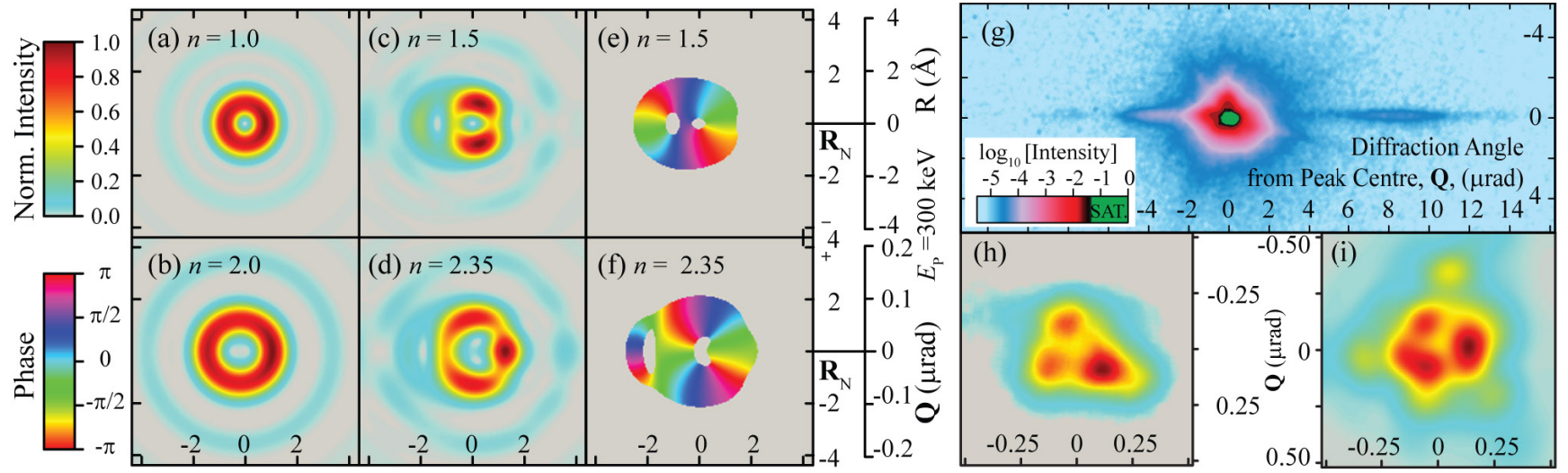

Figure 2. Calculated PSF intensity in the cases where $n=1,1.5,2$ and 2.35 (a, b, c and d respectively), and associated phase in the region containing $80 \%$ of the probe current for $n=1.5$ (e) and 2.35 (f) cases. Observed diffraction pattern from plate $(\mathrm{g})$ and central region of this pattern $(\mathrm{h})$. Including spherical, chromatic, three-fold aberration and convolving the demagnified source with the PSF helps the model pattern (i) agree with experiment, though it appears other aberrations need to be considered. 